\section{Looking time, stimulus complexity, and the perceptual deprivation effect*}

\author{
BRUCE T. LECKART, BRADLEY GLANVILLE, EDWARD HOOTSTEIN \\ KENNETH KELEMAN, and R. M. YAREMKO \\ San Diego State College, San Diego, Calif. 92115
}

Each of 29 college students viewed stimuli of low, middle, and high complexity under instructions to view each stimulus until they "finished looking at it." Perceptual deprivation periods of 2,16 , or $30 \mathrm{sec}$ preceded each stimulus. The results indicated that looking time was a direct function of deprivation level and stimulus complexity and that the two variables did not interact. An explanation in terms of the concept of an optimal level of arousal was suggested.

The last decade has seen an increased interest in human exploratory behavior. A significant body of literature in this area has been concerned with looking time, i.e., the time $S$ spends looking at a stimulus when $S$ controls stimulus offset. One line of research in this area has shown that looking time is a function of stimulus conditions preceding the viewing period. For example, it has been shown that looking time is a function of the number of stimulus exposures (Cantor \& Cantor, 1966; Leckart, 1966), the complexity of previously viewed stimuli (Tennison, $1968)$, and presentation rate of previously viewed stimuli (Leckart, Keeling, \& Bakan, 1966).

Recently, Leckart, Levine Goscinski, \& Brayman (1970) showed that looking time could be modified by administering brief periods of "perceptual deprivation" (a darkened experimental chamber) immediately before the viewing period. In that study, a direct relationship was obtained between duration of looking and deprivation intervals of 2-30 sec. Receptor adaptation and avoidance responding interpretations were ruled out, and it was concluded that short-term perceptual deprivation most likely produces a "need for stimulation" satisfied by viewing the stimulus for longer durations. Because of the potential significance of the relationship of these findings to those obtained in studies of long-term sensory restriction (e.g., Schultz, 1965), further study of the phenomenon was considered imperative. Since stimulus complexity is likely to be an important determinant of the need for stimulation, the present study was designed to obtain additional

*Supported in part by grants to the first and last authors from the San Diego State College Foundation. Requests for reprints should be addressed to Bruce $T$. Leckart, Department of Psychology, San Diego State College, San Diego, Calif. 92115. parametric information on the perceptual deprivation effect as a function of stimulus complexity. SUBJECTS

The Ss were 19 female and 10 male introductory psychology students at San Diego State College. They volunteered to satisfy a class requirement.

\section{APPARATUS}

Data were collected from the $S$ seated in a lightproof chamber $(1.8 \times 1.8 \mathrm{~m})$ and facing a $30 \times 30 \mathrm{~cm}$ rear-projection screen in one of the walls. The screen was at eye level and approximately $75 \mathrm{~cm}$ from S's face. When projected from a Kodak Carousel (Model 800) projector, stimuli were displayed at $20 \times 30 \mathrm{~cm}$. Wratten neutral-density filters on the lens reduced intensity of the display by $90 \%$. The stimuli were 27 different color slides of objects, arrays of objects, and landscapes from a group of 129 slides previously rated by 149 Ss on a 7-point scale of complexity (Leckart, 1966). The mean ratings of the stimuli were: low complexity, 6.36 ; middle complexity, 4.28; high complexity, 2.34. There were nine stimuli for each complexity level. A 28 th slide from the middle complexity range was used to start the series. Looking times were not recorded for this stimulus, since it was not preceded by any systematic deprivation period. $A$ hand button switch allowed $S$ to terminate a stimulus, and looking times were recorded to the nearest $0.1 \mathrm{sec}$.

\section{PROCEDURE}

A 3 by 3 within-Ss design was used in which the amount of perceptual deprivation preceding a stimulus (2, 16 , or $30 \mathrm{sec}$ ) was orthogonal to the three levels of stimulus complexity. Deprivation was defined as darkness occurring between S's termination of a stimulus and automatic onset of the next slide. The 27 experimental trials were divided into three blocks of nine trials each. Each block contained one of each of the nine conditions randomly reordered for each $\mathrm{S}$. Within each complexity level, the assignment of stimuli to conditions was reordered randomly for each $S$ to prevent stimulus by trials confounding. The Ss were told that the study was to investigate changes in skin resistance to visual stimuli. Sham finger electrodes were attached to S's left hand, and he was told that the leads were connected to a polygraph in the next room. The $S$ was told to view each stimulus until he was "finished looking at it" and then to terminate it by pressing the button.

\section{RESULTS}

For each $S$, mean looking times were computed for each of the nine conditions. These data are presented in Fig. 1. The figure indicates a clear positive relationship between stimulus complexity and looking time as well as a positive relationship between looking time and deprivation level. Analysis of variance revealed that the main effect for complexity was significant $[F(2,56)=31.25, p<.001]$, as was the deprivation variable $[F(2,56)=10.06, \quad p<.001]$. The Complexity by Deprivation interaction was not significant $(F<1.0)$.

$$
\text { DISCUSSION }
$$

These results are interesting for at least two reasons: first, they serve as a confirmation of the short-term perceptual deprivation effect reported by Leckart et al (1970) and demonstrate that this effect is not restricted to random geometric forms, such as used by Leckart et al, but occurs with "real world" stimuli such as color photographs of objects and landscapes; second, the significant complexity effect and the fact that the relationship between deprivation and looking time was essentially the same for all levels of complexity lend further support to the notion that a need for stimulation underlies the short-term perceptual deprivation effect.

The notion of a need for stimulation suggests an interpretation

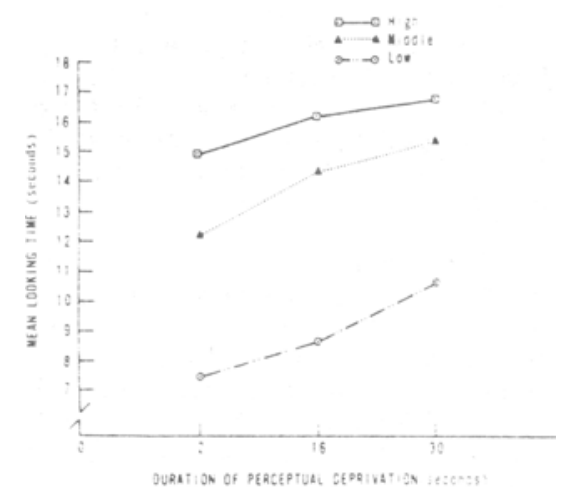

Fig. 1. Looking time as a function of stimulus complexity and perceptual deprivation. 
derived from the concept of organisms maintaining an optimal level of cortical arousal (e.g., Hebb, 1955). This position is based on the assumption that $\mathbf{S}$ obtains stimulus change as he scans the stimulus and that stimulus change produces arousal. When the opportunity to obtain stimulus change is denied by environmental conditions (such as by an enforced period of stimulus deprivation), S's arousal level descends from the optimal and continues to descend as deprivation continues. If one then assumes that the farther $S$ is from the optimal, the less stimulus change is needed to produce an increase in the level of arousal and that $\mathrm{S}$ continues to explore as long as the stimulus raises the level of arousal towards the optimal or maintains the optimal, the explanation of the current findings is straightforward. The long looking times that occur following long periods of deprivation stem from the ability of the stimulus to provide prolonged increases in the level of arousal because of S's distant position from the optimal. That is, when $S$ is far from the optimal, little stimulus change is needed to produce a given increase in arousal. Consequently, the arousal-producing potential of the stimulus is exhausted slowly. Similarly, the short looking times that occur following brief periods of deprivation stem from the inability of the stimulus to provide prolonged arousal because of S's close proximity to the optimal. That is, when $S$ is close to the optimal a large amount of stimulus change is needed to produce a given increase in arousal. Consequently, the arousal-producing potential of the stimulus is exhausted rapidly. The effects of stimulus complexity are explainable in a similar fashion: complex stimuli are looked at longer than simple stimuli because complex stimuli contain more arousal-producing properties and are therefore capable of either raising the arousal level or maintaining an optimal level for longer periods of tim $r$

\section{CANTOR, REFERENCES}

H., \& CANTOR, G. N. Functions relating children's observing behavior to amount and recency of stimulus familiarization. Journal of Experimental Psychology, 1966, 22. 859-863.

HEBB, D. O. Drives and the C. N. S. (conceptual nervous system). Psychological Review, 1955, 62, 243-254.

LECKART, B. T. Looking time: The effects of stimulus complexity and familiarity. Perception \& Psychophysics, 1966, 1, 142-144.

LECKART, B. T., KEELING, K. R., \& BAKAN, $P$. The effect of rate of presentation on free looking time. Perception \& Psychophysics, 1966, 1, 107-109.

LECKART, B. T., LEVINE, J. R., GOSCINSKI, C., \& BRAYMAN, W. Duration of attention: The perceptual deprivation effect. Perception \& Psychophysics, 1970, 7, 163-164.

SCHULTZ, D. P. Sensory restriction effects on behavior. New York: Academic Press, 1965. P. 145.

TENNISON, J. Duration of attention as a function of an adaptation to stimulus complexity. Unpublished dissertation, 1968, Ohio University.

\section{Illusion decrement in intersecting line figures*}

\author{
STANLEY COREN \\ The New School for Social Research, New York, N.Y. 10011 \\ and \\ JOAN S. GIRGUS
}

The City College of the City University of New York, New York, N.Y. 10031

This study tests the generality of illusion decrement using four illusion figures involving intersecting lines: the Zoellner, Poggendorff, Wundt-Hering, and Mueller-Lyer figures. Five minutes of inspection with testing at 1-min intervals led to significant decrement in all figures. The rate of decrement was the same for all figures. These data are consistent with a judgmental theory of geometric illusions.

Most theories of visual geometric illusions can be roughly categorized into one of two classes. The first class contends that visual illusions are created by peripheral distortion of the inputs (Chaing, 1968; Ganz, 1966; Békésy, 1967). The second class of theories suggests that judgmental

* We would like to thank Myra Agdern for her assistance in the collection of these data. variables provide the best explanation for the perceptual distortion (Carr, 1935; Piaget, 1969; Erlebacher \& Sekuler, 1969; Gregory, 1966).

Illusion decrement (i.e., the diminution of illusion magnitude with practice or inspection) is often the principal data offered in support of a central judgmental, as opposed to a peripheral optical or neural, mechanism (Over, 1968). Since a judgmental mechanism presumably reflects the manner in which an $O$ has learned to view certain stimuli in the past, it is fairly easy to imagine how such a mechanism could account for a decrement in illusion magnitude following inspection. It is somewhat more difficult to see how inspection might affect a physiological mechanism such as the optical or neural system in such a way that would lead to a decrement in illusion magnitude.

One major shortcoming of illusion decrement data is that it has been almost exclusively confined to the Mueller-Lyer figure, although some tentative evidence for decrement of the Poggendorff illusion has been offered by Cameron \& Steele (1905) and for decrement of a curvature illusion by Coren \& Festinger (1967). The occurrence of decrement in the magnitude of the Mueller-Lyer illusion is, however, well established (Judd, 1902; Lewis, 1908; Mountjoy, 1958; Day, 1962). Although the reasons for the decrease in illusion magnitude with practice are not yet clear, there are indications that information from erroneous saccadic eye movements made during inspection of the figure facilitates the process (Festinger, White, \& Allyn, 1968; Burnham, 1968).

Since decrement has been used as evidence for a judgmental mechanism in visual illusions, it is important to ascertain if decrement in illusion magnitude with inspection is a property of other illusion figures besides the Mueller-Lyer. The experiment reported below attempts to determine this for four illusion figures, each of which manifests a different type of distortion. One is an illusion of direction (the Zoellner), one an illusion of shape (the Wundt-Hering), one an illusion of displacement (the Poggendorff), and the last is an illusion of extent in which decrement is known to occur (the Mueller-Lyer). These particular illusions were chosen for investigation both because they manifest a variety of distortions and because they all contain intersecting line elements which are the optimal configurations for the operation of both optical aberrations (Chaing, 1968) and neural inhibitory interaction (Ganz, 1966; Békésy, 1967).

STIMULI AND APPARATUS

The illusion forms used in this experiment are represented in Fig. 1. The Zoellner Mlusion

The Zoellner illusion (Fig. 1A) was constructed with 2-mm-wide black lines. The three shafts were $11 \mathrm{~cm}$ long. Each of the intersecting lines was $3.5 \mathrm{~cm}$ long and crossed the shaft at an angle of $30 \mathrm{deg}$ from the vertical. The 